Präv Gesundheitsf 2010 · 5:298-299 DOI 10.1007/s11553-010-0269-7

Online publiziert: 24. Oktober 2010

(c) Springer-Verlag 2010

\section{A. Pieter}

Fachbereich Gesundheitsförderung, Deutsche Hochschule für Prävention

und Gesundheitsförderung, Hermann-Neuberger-Sportschule, Saarbrücken

\title{
Prävention - Was die Wirksamkeit erschwert und wie Prävention gelingen kann
}

Die Frage der Praxistauglichkeit von Programmen zur Prävention und Gesundheitsförderung setzt auf Rezipientenseite ihre prinzipielle Wirksamkeit voraus. Diese kann jedoch in vielen Fällen nicht eindeutig nachgewiesen und der Intervention genuin zugesprochen werden. Hierfür gibt es eine Vielzahl von Gründen. Neben der Frage, inwieweit Ort und Zeit der Interventionsdurchführung sowie der zeitliche Umfang der Intervention richtig gewählt wurden, spielen auch die persönlichen Kompetenzen der Teilnehmenden und Durchführenden eine wichtige Rolle. Weiterhin wird oftmals in diesem Kontext immer noch auf - teilweise therapeutische - Verfahren zurückgegriffen, die sich für die Umsetzung in der Präventionspraxis unter den dort vorherrschenden Gegebenheiten und Settings nur bedingt eignen. Des Weiteren ist die Compliance der Teilnehmenden zu berücksichtigen.

Die Autorinnen und Autoren in diesem Heft beleuchten diese Aspekte der prinzipiellen Wirksamkeit von Prävention aus den Blickwinkeln der unterschiedlichsten Disziplinen und liefern damit sowohl für die Forschung als auch für die Praxis wertvolle Hinweise bezüglich der Konzeption, Implementation und Evaluation von Interventionsmaßnahmen. Die ersten beiden Beiträge zielen auf das Profil der Teilnehmer an Präventionsmaßnahmen ab: A. Pieter et al. arbeiten in ihrem Beitrag heraus, von welchen Bedingungen es abhängt, ob sich ein Mensch aus rationalen Gründen und aus eigenem Antrieb für präventives und gesundheitsförderliches Verhalten entscheidet und wann es aus Sicht dieses Menschen sogar rational sein kann, sich nicht gesundheitsförderlich zu verhalten. B. Hagen et al. zeigen in ihrem Artikel, dass sich die Vorsorgequalität bei Teilnehmern des Disease-Management-Programms „koronare Herzkrankheiten“ über die Zeit zwar kontinuierlich verbessert, jedoch bei weitem nicht alle Patientengruppen in einem gleichen Ausmaß von der Maßnahme profitieren. Sie kristallisieren Patientenkollektive heraus, die im Rahmen der KHK-Prävention eine erhöhte Aufmerksamkeit verlangen. Vor dem Hintergrund der Diskussion um die Einführung eines Präventionsgesetzes in Deutschland beleuchtet J. Madarász die Historie des staatlichen Umgangs mit Herz-Kreislauf-Erkrankungen in drei Ländern zwischen 1945 und 1990. Die Autorin kommt zu dem Schluss, dass es in keinem der analysierten Länder überzeugend gelungen ist, Prävention nachhaltig im Gesundheitssystem zu implementieren und stellt diejenigen Aspekte heraus, die hierfür verantwortlich zeichnen.

Der Beitrag von A. Neumann und P. Schwarz thematisiert die Hindernisse bei der Implementation von Konzepten zur Prävention des Diabetes. Die Autoren weisen anhand eines Kommunikationsmodells und einer daraus resultierenden Strategie einen Weg auf, wie Prävention in diesem Bereich zukünftig wirksamer gestaltet werden kann. Auch H. Günay und K. Meyer berichten aus der Praxis und stellen ein prä- und postnatales Frühpräventionskonzept zur Verbesserung der Zahngesundheit von Müttern und Kindern dar. Abgeleitet aus der im Rah- men einer Längsschnittstudie überprüften Wirksamkeit der Maßnahme stellen die Autorinnen Möglichkeiten der Implementation zahnärztlicher Vorsorgeuntersuchungen während der Schwangerschaft und nach der Geburt vor, welche zum einen eine weitere Verbreitung des Frühpräventionskonzeptes bewirken und zum anderen auch Risikogruppen erreichen können.

Aus der Praxis des Modellprojekts Pro Kind berichten K. Adamaszek und R. Schneider. Sie stellen anschaulich dar, wie die Qualität dieses Hausbesuchsprogramms der „Frühen Hilfen“ durch geeignete Qualifizierungsmaßnahmen der Familienbegleiterinnen nachhaltig gefördert werden kann. Daran anknüpfend berichten V. Kurtz et al. erste positive Effekte des Projekts Pro Kind auf die selbsteingeschätzte mütterliche Selbstwirksamkeit und Kompetenz, wobei sie Verzerrungen bei der subjektiven Einschätzung nicht ausschließen können und kritisch diskutieren. Weiterhin geben die Autoren wertvolle Hinweise für das weitere praktische Vorgehen im Rahmen des Modellprojekts.

Um Präventionsmaßnahmen zielgruppenorientiert konzipieren zu können, ist es unerlässlich das Verhalten und die Wünsche der Teilnehmer zu kennen. Hierauf zielt der Beitrag von C. Groß et al. ab. Sie stellen die Ergebnisse des Forschungsprojekts VEGIS dar, in dessen Rahmen analysiert wurde, welche Freizeitangebote Kinder und Jugendliche innerhalb und außerhalb der Schule abhängig von Alter, Geschlecht und Adipositasdiagnose nut- 
zen und welche Wünsche sie diesbezüglich äußern. Aus den Befunden lassen sich konkrete Hinweise für die Konzeption von Sport- und Bewegungsangeboten, beispielsweise im Rahmen extracurricularer Schulaktivitäten, ableiten.

Eine Vielzahl von Präventionsmaßnahmen fokussiert immer noch alleinig auf die Wissensvermittlung als Interventionsmethode ab. M. Wolf berichtet Ergebnisse die zeigen, dass über eine bloße gesundheitliche Aufklärungskampagne keine Verhaltensmodifikation bei kardiovaskulären Risikopatienten zu erwarten ist. Die Autorin rekurriert vielmehr darauf, dass genderspezifische Unterschiede, beispielsweise hinsichtlich des Körper- und Selbstbildes oder subjektiver Theorien zu einer Risikopersönlichkeit, bei der Maßnahmenkonzeption eine Rolle spielen sollten. Barrieren und Potenziale bei der erfolgreichen Umsetzung eines Naschgartens stellen B. Blättner et al. dar. Es wird anschaulich gezeigt, dass die Wirksamkeit von Präventionsmaßnahmen nicht unwesentlich von moderierenden Faktoren, wie beispielsweise der gärtnerischen Fachkompetenz der Beteiligten oder dem partizipatorischen Umgang mit den teilnehmenden Kindern, beeinflusst wird. Ebenfalls aus dem Setting Schule berichten T. Kliche et al. In ihrem Beitrag werden zum einen die Schwierigkeiten sichtbar, auf die wissenschaftliche Begleitforschung im Kontext Schule trifft, zum anderen werden die Faktoren und Rahmenbedingungen herauskristallisiert, welche für die erfolgreiche Umsetzung von Settingprojekten in der Schule verantwortlich zeichnen.

Der Bedeutung der Bewertung des eigenen sozialen Status in der traditionellen Public-Health-Forschung, über die traditionell hierzu verwendeten Faktoren wie Bildung, Einkommen und berufliche Position hinaus, widmet sich der Beitrag von R. Hegar und A. Mielck. Sie zeigen, aufbauend auf den Ergebnissen eines systematischen Reviews, die Relevanz des subjektiven sozialen Status (SSS) hinsichtlich des Gesundheitszustandes, respektive dem Vorliegen gesundheitlicher Risikofaktoren. Sie leiten Handlungsempfehlungen für Forschung und Praxis ab, die es ermöglichen sollen, die Ausgangslage der jeweiligen Zielgruppe besser einzuschät- zen und Maßnahmen zielgruppenorientierter konzipieren zu können.

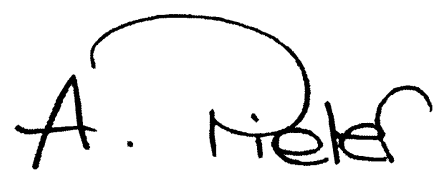

A. Pieter

\section{Korrespondenzadresse Prof. Dr. phil. A. Pieter}

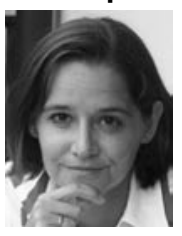

Fachbereich Gesundheitsförderung, Deutsche Hochschule für Prävention und Gesundheitsförderung, Hermann-NeubergerSportschule, 66123 Saarbrücken a-pieter@dhfpg.de

\section{Michael Terbille (Hrsg.) Münchener Anwaltshandbuch Medizinrecht.}

München:Verlag C. H. Beck 2009, 1282 S., (ISBN 978-3-406-56204-4), Leinen, 128.00 EUR

Die Bedeutung rechtlicher Fragen in der Medizin nimmt zu. Dies gilt nicht nur für den Komplex der ärztlichen Heilbehandlung und das Arzt-Patienten-Verhältnis, sondern auch für das institutionelle Gefüge und die Organisation der Krankenversorgung sowie Prävention und der öffentlichen Gesundheitsfürsorge. Die praktische Relevanz des Medizinrechts ist für Juristen und die nichtjuristischen Akteure im Gesundheitswesen in den letzten Jahren erheblich gestiegen. In diesem Handbuch wird die komplexe Querschnittsmaterie „Medizinrecht" dem Leser auf 13 Kapitel verteilt ausgebreitet. Die inhaltliche Spannweite dieser Kapitel erfasst die praxisrelevanten Teilgebiete des Medizinrechts. Den Anfang macht das zivilrechtliche Arzthaftungsrecht, dessen Darstellung breiten Raum einnimmt und mit nützlichen Praktikerhinweisen angereichert ist. Generell bildet das Arzthaftungsrecht zusammen mit seiner strafrechtlichen Dimension (Kapitel 2) und dem Medizinschadensrecht (Kapitel 11) einen thematischen Schwerpunkt des Handbuchs. Auch die weiteren Rechtsfragen, die mit der ärztlichen Berufsausübung verknüpft sind (Berufsrecht, Vertragsfragen, Gesellschaftsrecht, Vergütung), werden in eigenen Kapiteln behandelt. Komplementär geht das Werk auf das Krankenhausrecht und das Recht der Veterinäre ein. Daneben wird das Recht der privaten Krankenversicherung und die gesetzliche Krankenversicherung nebst den Grundzügen der Pflegeversicherung in jeweils eigenen Kapiteln dargestellt. Des Weiteren beleuchtet das Handbuch, wenn auch stellenweise knapp, auch die Grundzüge des Apotheken-, Arzneimittel- und Medizinprodukterechts.

Das umfangreiche und aktuelle Handbuch richtet sich an jeden im Medizinrecht tätigen Rechtsanwalt. Es wird darüber hinaus auch für juristisch tätige Mitarbeiter in Behörden, Krankenhäusern und bei Haftpflicht- und Krankenversicherern nützlich sein. Das Werk kann dieser Zielgruppe daher ohne Weiteres empfohlen werden. 